\title{
SCALING, STABILITY AND DISTRIBUTION OF THE HIGH-FREQUENCY RETURNS OF THE IBEX35 INDEX
}

\author{
PABLO SUÁREZ-GARCÍA AND DAVID GÓMEZ-ULLATE
}

\begin{abstract}
In this paper we perform a statistical analysis of the high-frequency returns of the IBEx35 Madrid stock exchange index. We find that its probability distribution seems to be stable over different time scales, a stylized fact observed in many different financial time series. However, an in-depth analysis of the data using maximum likelihood estimation and different goodness-of-fit tests rejects the Lévy-stable law as a plausible underlying probabilistic model. The analysis shows that the Normal Inverse Gaussian distribution provides an overall fit for the data better than any of the other subclasses of the family of the Generalized Hyperbolic distributions and certainly much better than the Lévy-stable laws. Furthermore, the right (resp. left) tail of the distribution seems to follow a power-law with exponent $\alpha \approx 4.60$ (resp. $\alpha \approx 4.28$ ). Finally, we present evidence that the observed stability is due to temporal correlations or non-stationarities of the data.
\end{abstract}

Keywords: financial time series, high-frequency returns, generalized hyperbolic distributions, Lévy-stable distributions, scaling laws, tail behaviour

\section{INTRODUCTION}

The marginal distribution of returns of financial assets have been placed under scrutiny since the times of Bachelier [5], and the idea of treating log-returns as independent identically distributed Gaussian random variables lies in the core of the most well-known and celebrated financial models [9, 37] and so it is crucial for derivative pricing and risk management. And even though this idea works fine as a first approximation, it is well documented that empirical financial data drawn from very different markets, time periods and instruments do not fit the Gaussian model [15, 20, 33, 38]. The empirical distributions of log-returns present tails heavier than Gaussian as well as many other non-trivial statistical properties collectivelly know as stylized facts [14] that place the Gaussian hypothesis in jeopardy and point towards a possible universal behavior of the underlying processes.

One of the most celebrated of these stylized facts is the scaling symmetry or stability of the distribution of log-returns, i.e. its invariance under aggregation up to rescaling. For independent identically distributed random variables the Gaussian law is the only distribution with finite second moment that has this property, and that is why the central limit theorem singles it out as the limiting distribution of rescaled sums of i.i.d. random variables with finite variance [11]. To observe stability in distributions other than Gaussian the requirement of a finite second moment has to be dropped. This seminal idea was first expounded in finance by Mandelbrot [33] who proposed the family 
of Lévy-stable laws as an alternative to the Gaussian model of log-returns. One feature of these probability distributions is the divergence of their second moment caused by the power-law behavior of its tails with characteristic exponent $\alpha<2$. Considering a financial market as a complex system of interacting agents in which prices are the outcome of many independent individual decisions, according to the generalized central limit theorem its limiting distribution should be a member of the Lévy-stable family - of which the Gaussian distribution is just a special case - with the normalization constant depending on the tail index of the power-law [11].

Therefore, it seems natural to investigate the tails of the distribution of log-returns in order to shed some light on its stability properties. However, this is a moot point: although some authors [20,33] have reported power-law behavior with $\alpha<2$, others have reported distributions with power-law tails far away from the stability regime [21, 23, 29, 30, 40]. It could be argued that there is an endemic arbitrariness of the leastsquare regression used to study the power-law behaviour in empirical data, but that problem could be overcome replacing it with maximum likelihood estimation together with goodness-of-fit techniques $[13$. This fact notwithstanding, it is also well known that an estimated tail index above two is not an evidence against stability: it could well have been produced by a stable distribution with $\alpha$ as low as 1.65 with the situation getting worse as we approach the $\alpha=2$ limit [16,36]. And to round this off, it is not only difficult to discriminate between different power-laws or even between stability or the lack of it; the sole task of distinguishing a power-law from a stretched exponential is still subject to debate [32], since certain empirical distributions of log-returns seem to decay asymptotically slower than any power-law 15, 24, 28.

Among the distributions with tails lighter than power-laws, a family that has been used with success to model log-returns are the Generalized Hiperbolic laws. The embryo of this family of distributions is the Hyperbolic distribution, first proposed by Ralph Alger Bagnold [6] to model the size distribution of the wind-blown sand. Later, Barndorff-Nielsen - still with the problem of the distribution of particle size in mind generalized it to the family of Generalized Hyperbolic $(G H)$ distributions [7], of which the hyperbolic distribution is a special case. Different subclasses of this family have been since then proposed as alternatives to both Gaussian and Lévy-stable laws as statistical models of financial returns, namely, the Skewed Student's $t$ distribution [10, 41], the Hyperbolic distribution by Eberlein and Keller [19,27, the Variance-Gamma of Madan and Seneta [31] and the Normal Inverse Gaussian (NIG) by Barndorff-Nielsen [8]. One of the most appealing properties of this family is its tail behavior, which is a power-law modulated by an exponential. These lighter tails seem well suited to fit the empirical distributions of log-returns as the studies cited above show, since the data seem to have tails heavier than Gaussian but still lighter than the Lévy-stable laws.

As can be inferred, the question of the true nature of the distribution of log-returns (or even its tail behavior) and the origin of its apparent stability is far from being settled, and therefore, the study of diverse financial time series drawn from different instruments, markets and time periods is pertinent in order to shed some light on this issue. In this paper we will carry out a thorough study of the distributional properties of the high-frequency log-returns of the index IBEx35 from the Madrid Stock Exchange. 
After reviewing the basic properties of both the Lévy-stable and Generalized Hyperbolic laws in Section 2, we will perform a series of fits of these families to the observed logreturns as well as different statistical tests to quantify their goodness-of-fit (Section 3). There, we will also study in close detail the tail behavior of the data in order to elucidate its possible stability and we will address the question of the scaling symmetry of the data. In Section 4 we will sum up the results of the previous section and we will confront them to those obtained in other studies. The conclusions will be expounded in Section 5 .

\section{LÉVY-STABLE LAWS AND GH DISTRIBUTIONS}

2.1. Lévy-stable laws. Lévy-stable laws do not have a closed analytical form for its probability density function in general, but they can be readily defined in terms of their characteristic function $\varphi(t)$ :

$$
\begin{gathered}
\varphi(t)=\exp \left[i t \mu-|\delta t|^{\alpha}(1-i \beta \operatorname{sgn}(t) \Phi)\right] \\
\Phi=\left\{\begin{aligned}
\operatorname{tg} \frac{\pi \alpha}{2} & \text { if } \alpha \neq 1 \\
-\frac{2}{\pi} \log |t| & \text { if } \alpha=1
\end{aligned}\right.
\end{gathered}
$$

The characteristic exponent $\alpha \in(0,2]$ determines the weight of the tails and the skewness parameter $\beta \in[-1,1]$ its asymmetry. The parameters $\mu$ and $\delta$ are its location and scale parameters respectively. The Gaussian distribution is a special case of this family with $\alpha=2, \beta=0$. A random variable $X$ is the limit in distribution of normalized sums of i.i.d random variables if and only if $X$ has a Lévy-stable law [11].

2.2. Generalized Hiperbolic laws. The Generalized Hyperbolic distribution can be parametrized in several ways. Following Prause [42], its probability density function can be written as:

$$
f(x ; \lambda, \delta, \alpha, \mu, \beta)=\frac{(\gamma / \delta)^{\lambda}}{\sqrt{2 \pi} K_{\lambda}(\delta \gamma)} e^{\beta(x-\mu)} \frac{K_{\lambda-1 / 2}\left(\alpha \sqrt{\delta^{2}+(x-\mu)^{2}}\right)}{\left(\sqrt{\delta^{2}+(x-\mu)^{2}} / \alpha\right)^{1 / 2-\lambda}}
$$

where $\gamma=\sqrt{\alpha^{2}+\beta^{2}}$ and $K_{\lambda-1 / 2}$ is the modified Bessel function of the third kind with index $\lambda-1 / 2$. The parameter $\alpha>0$ determines the shape of the distribution and $0 \leq|\beta|<\alpha$ its skewness. The usual location and scale parameters are $\mu$ and $\delta$. The parameter $\lambda$ characterizes certain subclasses and influences the size of the mass contained in the tails. These distributions can be thought as mean-variance mixtures of Gaussian distributions where the mixing distribution is the Generalized Inverse Gaussian distribution [18].

Letting $\lambda=-\frac{1}{2}$ we obtain the Normal Inverse Gaussian distribution (NIG), whose probability density function is: 


$$
f(x)=\frac{\alpha \delta K_{1}\left(\alpha \sqrt{\delta^{2}+(x-\mu)^{2}}\right)}{\pi \sqrt{\delta^{2}+(x-\mu)^{2}}} e^{\delta \gamma+\beta(x-\mu)}
$$

All its moments are well defined since it decays as $x^{\alpha} e^{-\beta x}$. The $N I G$ distribution is the only subclass of the $G H$ family which is closed under convolution; this fact greatly simplifies the computations for option pricing [17].

Letting $\lambda=-\frac{\nu}{2}$ and $\alpha \rightarrow|\beta|$ in the formula 3 above, we obtain the GH skew Student's $t$ distribution. Its density is given by:

$$
f(x)=\frac{\left.2^{\frac{1-\nu}{2}} \delta^{\nu}|\beta|^{\frac{1+\nu}{2}} K_{\frac{\nu+1}{2}}\left(\sqrt{\beta^{2}\left(\delta^{2}+(x-\mu)^{2}\right.}\right)\right) e^{\beta(x-\mu)}}{\Gamma\left(\frac{\nu}{2}\right) \sqrt{\pi}\left(\sqrt{\delta^{2}+(x-\mu)^{2}}\right)^{\frac{\nu+1}{2}}}
$$

This is the only GH subfamily with different asymptotic behaviour of its density function: one tail is a power-law with characteristic exponent equal to $-\nu / 2-1$ and the other is a power-law with exponent $-\nu / 2-1$ modulated by a factor $e^{-2|\beta||x|}$. If the asymmetry parameter $\beta$ is zero, we recover the classical Student's $t$ distribution with symmetric and power-law tails, with a well defined second moment for $\nu>2[1]$.

\section{AnAlysis of the DATA}

3.1. The data. Our data set contains the price ticks of the index IBEx35 of the Madrid Stock Exchang 1 . The index IBEx35 is a weighted index formed by the 35 most liquid Spanish stocks traded at the Madrid Stock Exchange. The data set covers the period from January 2nd 2009 to December 31st 2010 and comprises 510 market days.

The values of the index are not updated evenly; the records oscillate between two and around twelve seconds. In order to have a well defined time interval we have sampled these ticks in fifteen-seconds intervals obtaining a series with 1036321 records. From this time series we have obtained the log-returns. However, some issues had to be taken into account before doing this. First, we have to discard the discontinuity created overnight to avoid artifacts: we therefore focus exclusively on intraday returns. Second, there is a 30 second uncertainty in the closing time of the session in order to avoid arbitrages: we have accordingly taken a security margin finishing our sessions at 17:29. Some authors [32] have also pointed out that the volatility pattern present during the day (the "lunch effect"; see Figure 1) should be taken into account by normalizing each return with the average absolute return of that time of the session. However, as happened in the study cited above, in ours we have not observed substantial differences between the raw and the normalized data; therefore, we have worked exclusively with raw returns. The final return series contains 1035810 records, with 2031 records for each market day (Figure 2). The sample statistics can be found in Table 1 once normalized in scale and location.

\footnotetext{
${ }^{1}$ Data obtained from www.tickdata.com.
} 


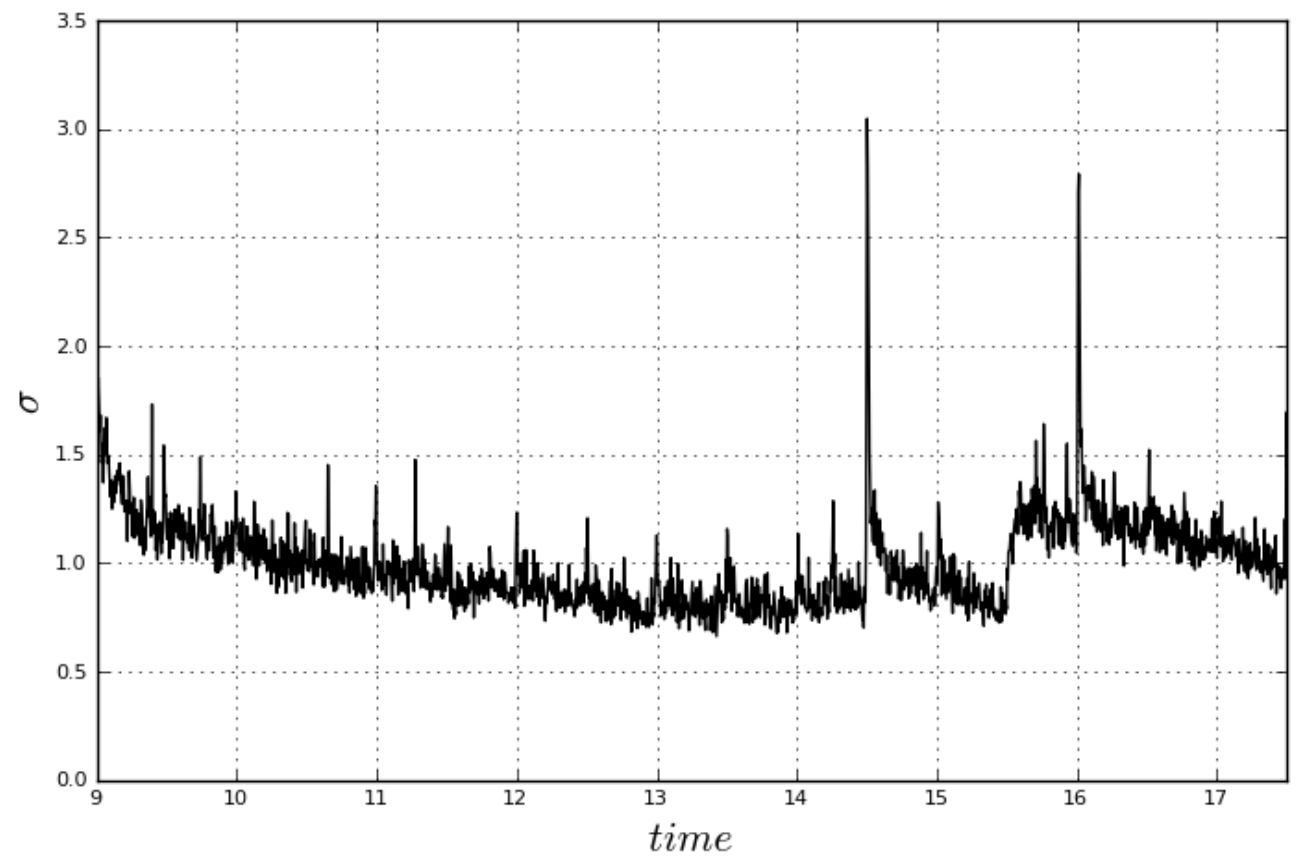

Figure 1. Lunch effect. At 15:30 CET Wall Street opens, and at 14:30 CET and 16:00 CET macroeconomic indicators in the USA are announced.

\begin{tabular}{cccccc}
\hline MAX. & $\min$. & $\mu$ & $\sigma^{2}$ & $\beta$ & $\kappa$ \\
\hline 29.181 & -28.184 & 0.000 & 1.000 & -0.241 & 13.659 \\
\hline
\end{tabular}

TABLE 1. Sample statistics.

3.2. Estimation of the parameters. Estimation of the parameters of all the distributions has been accomplished using the method of maximum likelihood. The asymptotic properties and optimality of this method of estimation are widely acknowledged [43]. However, for the family of Lévy-stable laws, parameter estimation via maximum likelihood is not straightforward due to the fact that an analytical expression of the probability density function is not available, and therefore, the method is only applicable by numerical approximation which is very time consuming due to the sample size. Other faster possibilities include methods based on the sample quantiles [35] or regression via the sample characteristic function; see [46] for a survey of the most usual estimation methods for this family of distributions. The estimated parameters can be found in Table 2, and Figure 3 shows the semilog plots of the estimated densities and the empirical histogram. 


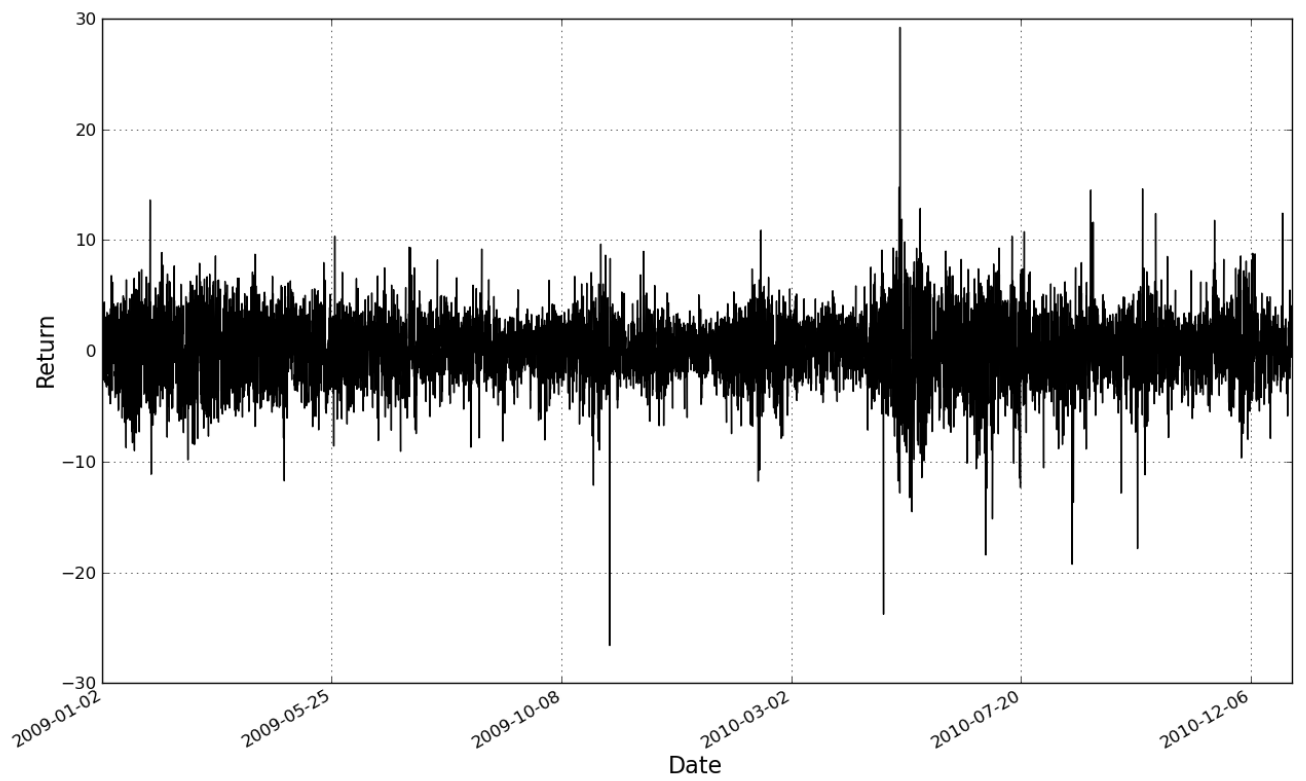

FiguRE 2. IBEX35 normalized logarithmic returns.

\begin{tabular}{lcccccc}
\hline Parameters: & $\mu$ & $\delta$ & $\beta$ & $\alpha$ & $\nu$ & $\lambda$ \\
\hline Lévy-stable & 0.0071 & 0.4825 & 0.0102 & 1.5358 & - & - \\
$G H$ & 0.0101 & 0.6495 & -0.0103 & 0.6296 & - & -0.5352 \\
Student's $t$ & 0.0101 & 0.9643 & -0.0089 & - & 2.7029 & - \\
NIG & 0.0101 & 0.6365 & -0.0103 & 0.6490 & - & - \\
\hline
\end{tabular}

TABLE 2. Estimated distribution parameters.

3.3. Goodness-of-fit tests. To quantify the goodness-of-fit of the estimated distributions three different statistical tests have been used: the $\chi^{2}$, the Kolmogorov-Smirnov and the Anderson-Darling [4] tests. These last two tests - based on the cumulative distribution function rather than on the probability density function as the simpler $\chi^{2}$ test - make a better use of the information contained in the sample since it does not need to be binned. Their drawback, however, is that they are much more computationally intensive since the distribution function has to be evaluated at the sample points and this implies millions of numerical integrations of trascendental functions. Apart from this, only when the parameters are part of the hypothesis the large-sample distribution of the test statistic is known; for estimated parameters (like in this case), this distribution is not known except for a few special cases [45]. Montecarlo simulation - the usual approach to tackle this problem [46 — is not feasible here due to the 

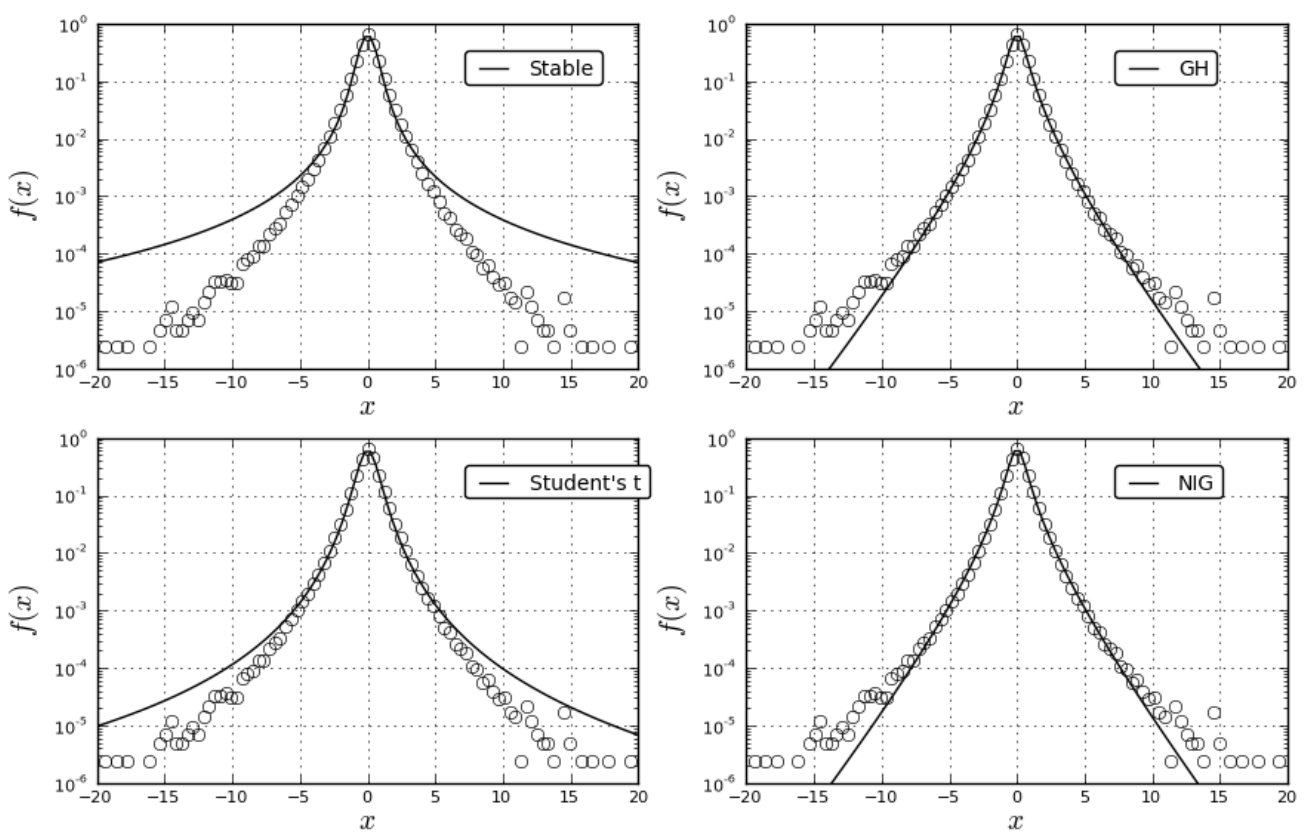

FIGURE 3. Histogram and estimated pdfs.

sample size and to the associated computational time needed to obtain the distribution function. Anyway - as Anderson [3] points out - the percentage points for the tests when the parameters are estimated are much smaller than those obtained when the parameters are known: a rejected hypothesis using these latter percentage points will then also be rejected with an even higher confidence level when using the former. In any case - and as a general rule - the lower the value of the test statistic, the better the fit.

\begin{tabular}{lccc}
\hline Test statistic & $\chi^{2}$ & K-S & A-D \\
\hline Lévy-stable & 17133.87 & 0.0175 & 512.17 \\
GH & 4556.36 & 0.0147 & 53.88 \\
Student's $t$ & 6967.21 & 0.0148 & 193.73 \\
NIG & 4911.18 & 0.0147 & 52.30 \\
\hline
\end{tabular}

TABLE 3. Goodness-of-fit statistics.

The values of the test statistics obtained for our data and the upper bounds for the critical points of the tests for the given confidence levels are shown in Tables 3 and 4. As it can be observed, the null hypothesis is rejected for any given reasonable confidence level for all the distributions. However, the hyperbolic distributions clearly outperform the Lévy-stable law. 


\begin{tabular}{cccc}
\hline Confidence & $\chi^{2}$ & K-S & A-D \\
\hline $5 \%$ & 231.92 & 0.00134 & 0.4614 \\
$1 \%$ & 245.48 & 0.00160 & 0.7435 \\
\hline
\end{tabular}

TABLE 4. Critical points for the goodness-of-fit tests.

For the members of the $G H$ family, a likelihood ratio $(\Lambda)$ test has been also performed. This will allow us to quantify which of the two subclasses (i.e. NIG or Student's $t$ ) is the soundest and whether or not the $G H$ model can be reduced to one of its subfamilies. The values obtained for the statistic $-2 \log \Lambda$ are tabulated in Table 5 along with the $p$ values of a $\chi_{1}^{2}$ variable, its large-sample distribution under the hypothesis of asymptotic normality of the maximum likelihood estimators. According to this, if we accept the hypothesis that the data follows a $G H$ distribution, we cannot reject with a confidence level of $2 \%$ the hypothesis that it in fact follows a $N I G$ distribution, while the hypothesis that the data follows a Student's $t$ distribution is rejected at any reasonable confidence level?

\begin{tabular}{ccc}
\hline Distribution & $-2 \log \Lambda$ & $\boldsymbol{p}$-value \\
\hline NIG & 5.49 & 0.02 \\
Student's $t$ & 4551.78 & $<10^{-16}$ \\
\hline
\end{tabular}

TABLE 5. Likelihood-ratio statistics for the $G H$ subfamilies

3.4. Asymptotic behavior. Since all of the usual distributions are rejected as plausible hypothesis for the data, we have also studied in detail the asymptotic behavior of the tails. On a log-log plot (Figure 4) they seem to fit rather well a straight line. Using the methodology proposed in [13] to analyze and estimate power-laws in empirical data, we have obtained a value of 4.60 (resp. 4.28) for the characteristic exponent $\alpha$ and a a value of 7.76 (resp. 6.70) for the scale parameter $x_{\min }$ for the right (resp. left) tail.

Even though the tails seem to follow a power-law well outside the stability region, a robust test to reject the hypothesis of stability or even of an exponential behavior is needed. It is well known that for moderate sample sizes an observed tail-index well above two cannot be used as an evidence against stability since it is highly unreliable estimator; if the distribution was really stable, an estimation of the tail parameter using the full sample by maximum likelihood would be more pertinent [36].

3.5. Stability of the data. According to the results of the last paragraph and considering the sample size, the most plausible hypothesis is the lack of stability of the distribution of returns. However, sampling the returns at different time scales $t$ - from

\footnotetext{
${ }^{2}$ We also performed all the tests for the Hyperbolic and Variance-Gamma distributions which yielded even worse $p$-values than those obtained for the Student's $t$. We thus decided not to include them among our results.
} 

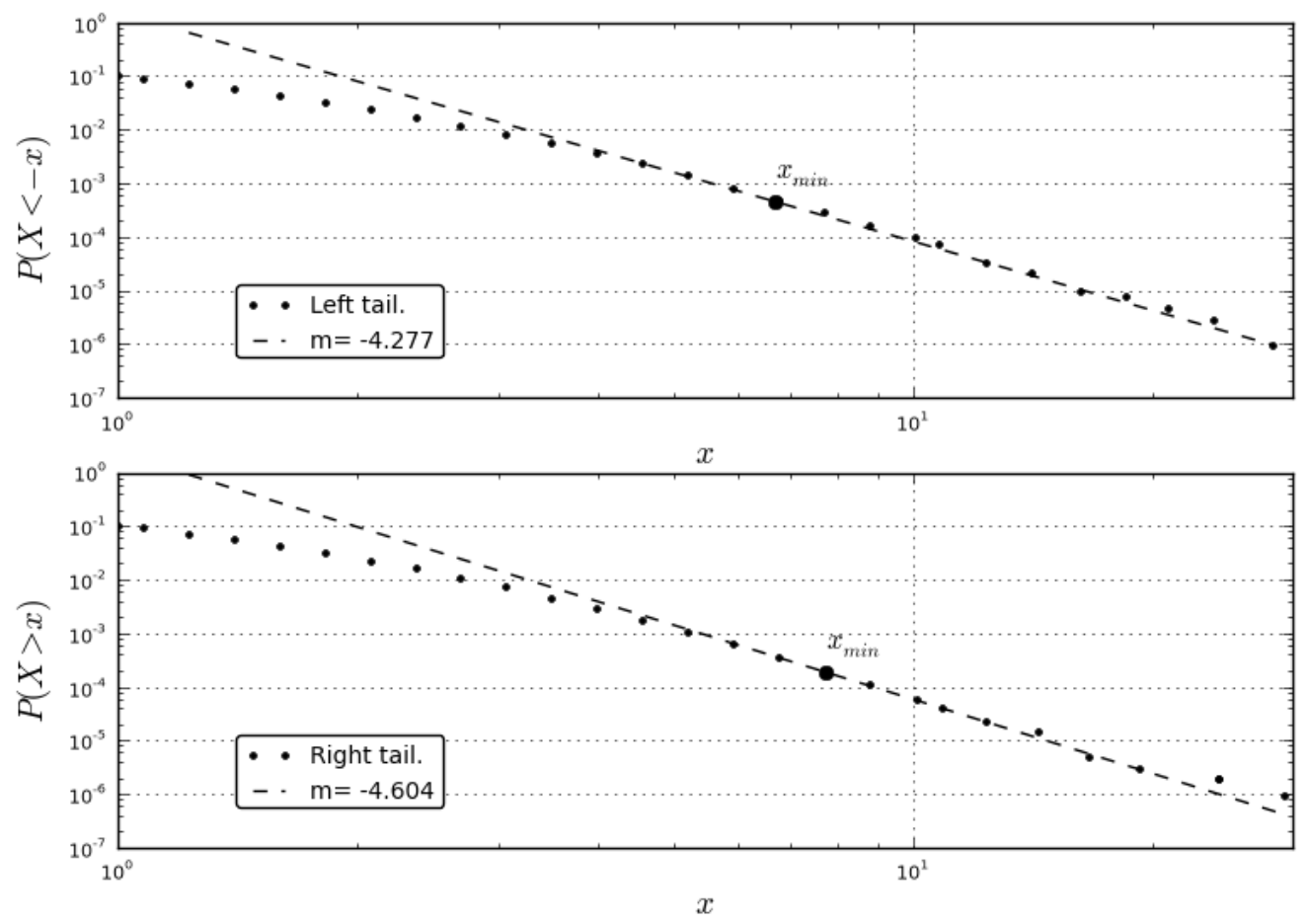

Figure 4. Tails of the complementary cumulative distribution function.

fifteen seconds up to half a day - and rescaling it with $t^{1 / 2}$ its distribution seems to remain stable ${ }^{3}$ (Figure 5, top panel). This suggests that this symmetry must therefore be the result of the presence of long memory in the data or to the temporal dependence of the parameters.

To support this facts, a reshuffling of the data has been performed (Figure 5, middle panel). It can be readily observed that Gaussianity is reached in a few minutes, as could be expected from the central limit theorem. Finally, we have also performed a daily reshuffling of the returns to verify if this scaling symmetry could be an artifact of the lunch effect. As can be observed in Figure 5 (bottom panel), the scaling symmetry of the data still holds, a fact that points towards long-range correlations as the most plausible explanation for this symmetry. It goes without saying that the long memory exhibited by the data and its autocorrelations deserve an in-depth study that shall be addresed in future work.

\footnotetext{
${ }^{3}$ The value of $1 / 2$ for the scaling exponent was obtained by Detrended Fluctuation Analysis, and it is the one expected for independent identically distributed random variables with finite second moment.
} 

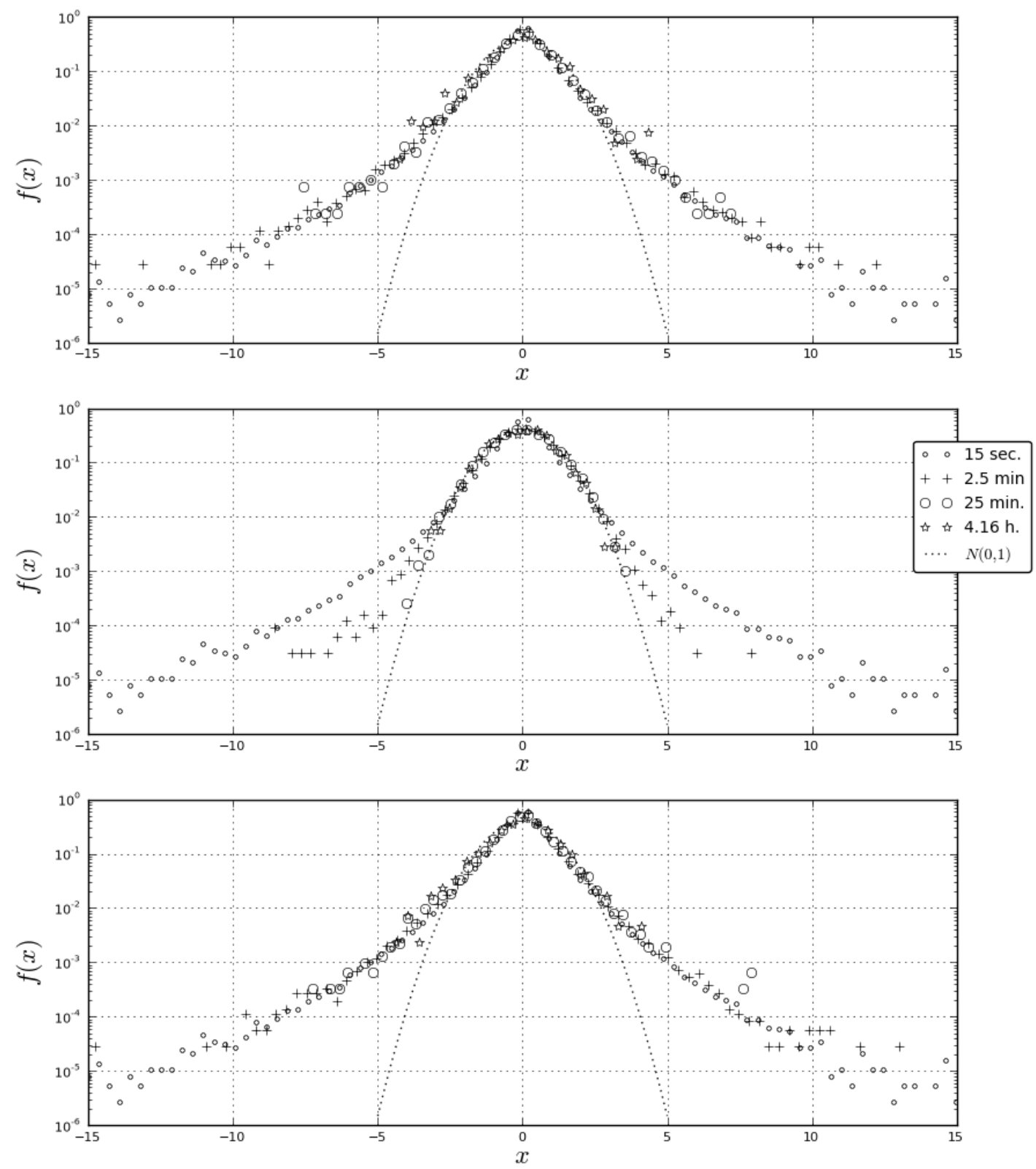

Figure 5. Rescaled probability distributions of the IBEX35 index observed at different time intervals (dotted line: $N(0,1)$ ). Top: raw data. Middle: reshuffled data. Bottom: daily reshuffled data. 


\section{Discussion}

As far as we know, this is the first study of the high-frequency returns of the IBEx35, so we will necessarily compare our results with those obtained for similar market indexes.

Platen and Sidorowicz, in their study of several world stock indexes [39], found that for the daily returns of the Madrid stock exchange the best fit was provided by a Student's $t$ with 4.51 degrees of freedom, while the $N I G$ distribution fit was not as sound. The same results were obtained for a broad group of world stock indexes as an extension of a previous study [25]. This result is in stark contrast with our findings, where the NIG distribution outperforms the other members of the $G H$ family as well as the Lévy-stable laws. As a matter of fact, according to the likelihood-ratio test, the five-parameter $G H$ family could be reduced to the four-parameter NIG model without much loss.

The Lévy-stable distributions seem to be also very well suited to model the log-returns of many stock market indexes: this is the case e.g. of the daily returns of the Hong Kong Hang SEng index. Further, for this index the Lévy-stable law provides a much better fit than the $N I G$ distribution [12]. Similar results have been observed for the IPC mexican index: the hypothesis of stability could not be rejected at $5 \%$ confidence level while the hypothesis of $N I G$ distributed daily log-returns was clearly rejected [2]. However, according to our findings, the Lévy-stable model is not the best option for modeling the high-frequency returns of the IBEX35 since the NIG distribution is a much better candidate.

Considering the tail behaviour of the distribution of log-returns, it is documented that the S\&P500 index follows a power law with $\alpha \approx 3[22,23]$, while the characteristic exponent of the German DAX lies in the range between 3 and 4 [30]. This is the most commonly accepted range for the characteristic exponent of the tails of the distribution of log-returns. However, the consensus is not complete, and some authors [26, 32] claim that a characteristic exponent in the range $\alpha \in[3,5]$ could be expected, and even that the decay could well be exponential rather than hyperbolic. We have obtained a seeming power-law behavior for the right (resp. left) tail of the distribution with exponent $\alpha \approx 4.60$ (resp. $\alpha \approx 4.28$ ), inside the accepted $\alpha \in[3,5]$ range. The only probability distribution analyzed in our study that could have this asymptotic behavior is the Student's $t$. The overall fit, however, rules it out as a plausible model.

The scaling invariance of the financial time series was first proposed and exploited by Mandelbrot in his investigation of the variations of cotton prices [33. In what regards to stock market indexes, scaling has been observed in the high-frequency returns of S\&P500 index 34 and in the OBX index of the Oslo stock exchange [4] among others. In both cases the aggregated log-returns were rescaled with $n^{-1 / \alpha}$, being the estimated tail parameter for the Lévy-stable fit (1.4 for the S\&P500 and 1.64 for the OBX, values that are similar to what we have estimated (1.53)). In our study, however, rescaling using the estimated tail exponent for the Lévy-stable fit destroys the symmetry; a value of $1 / \alpha=0.5$ obtained by $D F A$ - and the one expected for finite second moment random variables - was used instead. 


\section{Summary}

We have performed a statistical analysis of the high frequency log-returns of the IBEx35 index of the Madrid stock exchange over a two year period (2009-2010). Particular attention has been paid to describing the best probability distribution for the data since this question is still controversial in the recent literature, the only fact commonly accepted (although not yet fully incorporated into pricing models) is the departure from normality. Our results show that among the members of the family of Generalized Hyperbolic laws the Normal Inverse Gaussian distribution is the one that provides the best fit for the data. Furthermore, the 5-parameter $G H$ family could be well reduced to the 4-parameter $N I G$ family without significant loss. This distribution also clearly outperforms the Lévy-stable laws as a statistical model for this index.

The tails of the distribution of log-returns behave as power laws with exponents $\alpha \approx 4.28$ (left tail) and $\alpha \approx 4.60$ (right tail), a fact that according to the generalized central limit theorem would not be compatible with the stability of the distribution under aggregation. However, the empirical distribution of log-returns has been observed to be stable over several time scales, ranging from a few seconds up to a few hours. We conjecture that time correlations among the data are probably responsible for this observed stability, since reshuffling the data destroys these time correlations and restores the expected convergence results predicted by the central limit theorem. A more thorough analysis of these time correlations shall be conducted in future work, together with the development of derivative pricing models that take into account more realistic distributions for the underlying assets.

\section{REFERENCES}

[1] K. Aas and I.H. Haff, The generalized hyperbolic skew Student's t-distribution, Journal of Financial Econometrics 4 (2006), no. 2, 275.

[2] L. Alfonso, R. Mansilla, and C.A. Terrero-Escalante, On the scaling of the distribution of daily price fluctuations in the mexican financial market index, Physica A: Statistical Mechanics and its Applications 391 (2012), 2990-2996.

[3] T. W. Anderson, Anderson-Darling tests of goodness-of-fit, International Encyclopedia of Statistical Science, Springer, 2011.

[4] T.W. Anderson and D.A. Darling, A test of goodness of fit, Journal of the American Statistical Association 49 (1954), no. 268, 765-769.

[5] L. Bachelier, Théorie de la spéculation, Gauthier-Villars, 1900.

[6] R.A. Bagnold, The physics of blown sand and desert dunes, Methuen, 1941.

[7] O. Barndorff-Nielsen, Exponentially decreasing distributions for the logarithm of particle size, Proceedings of the Royal Society of London A: Mathematical and Physical Sciences 353 (1977), no. $1674,401$.

[8] O.E. Barndorff-Nielsen, Normal inverse gaussian distributions and the modeling of stock returns, Research report 300 (1995).

[9] F. Black and M. Scholes, The pricing of options and corporate liabilities, The journal of political economy 81 (1973), no. 3, 637-654.

[10] R.C. Blattberg and N.J. Gonedes, A comparison of the stable and student distributions as statistical models for stock prices, The Journal of Business 47 (1974), no. 2, 244-280.

[11] L. Breiman, Probability, Addison-Wesley, 1968.

[12] K. Burnecki, J. Gajda, and G. Sikora, Stability and lack of memory of the returns of the Hang Seng index, Physica A: Statistical Mechanics and its Applications 390 (2011), 3136-3146. 
[13] A. Clauset, C.R. Shalizi, and M.E.J. Newman, Power-law distributions in empirical data, Arxiv preprint arXiv:0706.1062 (2007).

[14] R. Cont, Empirical properties of asset returns: stylized facts and statistical issues, Quantitative Finance 1 (2001), no. 2, 223-236.

[15] R. Cont, M. Potters, and J.P. Bouchaud, Scaling in stock market data: stable laws and beyond, CNRS Workshop on Scale Invariance, Les Houches (Graner Dubrulle and Sornette, eds.), 1997.

[16] W.H. DuMouchel, Estimating the stable index $\alpha$ in order to measure tail thickness: a critique, The Annals of Statistics 11 (1983), no. 4, 1019-1031.

[17] E. Eberlein, Application of generalized hyperbolic lévy motions to finance, Lévy processes: theory and applications (O.E. Barndorff-Nielsen, T. Mikosch, and S.I. Resnick, eds.), Birkhauser, 2001.

[18] E. Eberlein and E. Hammerstein, Generalized hyperbolic and inverse Gaussian distributions: limiting cases and approximation of processes, Seminar on Stochastic Analysis, Random Fields and Applications IV, vol. 58, Centro Stefano Franscini, Ascona, 2002, pp. 221-264.

[19] E. Eberlein and U. Keller, Hyperbolic distributions in finance, Bernoulli 1 (1995), no. 3, 281-299.

[20] E.F. Fama, The behavior of stock-market prices, The journal of Business 38 (1965), no. 1, 34-105.

[21] J. D. Farmer, Physicists attempt to scale the ivory towers of finance, Computing in Science \& Engineering 1 (1999), no. 6, 26-39.

[22] P. Gopikrishnan, M. Meyer, L.A.N. Amaral, and H.E. Stanley, Inverse cubic law for the distribution of stock price variations, The European Physical Journal B-Condensed Matter and Complex Systems 3 (1998), no. 2, 139-140.

[23] P. Gopikrishnan, V. Plerou, L.A.N. Amaral, M. Meyer, and H.E. Stanley, Scaling of the distribution of fluctuations of financial market indices, Physical Review E 60 (1999), no. 5, 5305.

[24] C. Gourieroux and J. Jasiak, Truncated maximum likelihood, goodness of fit tests and tail analysis, Sonderforschungsbereich 373, 1998.

[25] S.R. Hurst and E. Platen, The marginal distributions of returns and volatility, L1-Statistical Procedures and Related Topics, IMS Lecture Notes-Monograph Series, vol. 31, 1997, pp. 301314.

[26] S. Kinsella and F. O'Brien, Maximum likelihood estimation of stable paretian distribution applied to index and option data, Proceedings of the INFINITI Conference on International Finance, 2009, pp. 8-9.

[27] U. Küchler, K. Neumann, M. Sørensen, and A. Streller, Stock returns and hyperbolic distributions, Mathematical and Computer Modelling 29 (1999), no. 10-12, 1-15.

[28] J. Laherrere and D. Sornette, Stretched exponential distributions in nature and economy: "fat tails" with characteristic scales, The European Physical Journal B: Condensed Matter and Complex Systems 2 (1998), no. 4, 525-539.

[29] T. Lux, The stable paretian hypothesis and the frequency of large returns: an examination of major german stocks, Applied financial economics 6 (1996), no. 6, 463-475.

[30] — The limiting extremal behaviour of speculative returns: an analysis of intra-daily data from the Frankfurt Stock Exchange, Applied Financial Economics 11 (2001), no. 3, 299-315.

[31] D.B. Madan and E. Seneta, The variance gamma (VG) model for share market returns, Journal of Business 63 (1990), no. 4, 511-524.

[32] Y. Malevergne, V. Pisarenko, and D. Sornette, Empirical distributions of stock returns: between the stretched exponential and the power law?, Quantitative Finance 5 (2005), no. 4, 379-401.

[33] B. B. Mandelbrot, The variation of certain speculative prices, The Journal of Business 36 (1963), no. 4, 394-419.

[34] R.N. Mantegna and H.E. Stanley, Scaling behaviour in the dynamics of an economic index, Nature 376 (1995), no. 6535, 46-49.

[35] J.H. McCulloch, Simple consistent estimators of stable distribution parameters, Communications in Statistics: Simulation and Computation 15 (1986), no. 4, 1109-1136.

[36] _ Measuring tail thickness to estimate the stable index $\alpha$ : a critique, Journal of Business \& Economic Statistics (1997), 74-81. 
[37] R.C. Merton, Theory of rational option pricing, The Bell Journal of Economics and Management Science 4 (1973), no. 1, 141-183.

[38] A. Pagan, The econometrics of financial markets, Journal of empirical finance 3 (1996), no. 1, $15-102$.

[39] E. Platen and R. Rendek, Empirical evidence on Student-t log-returns of diversified world stock indices, Journal of statistical theory and practice 2 (2008), no. 2, 233-251.

[40] V. Plerou, P. Gopikrishnan, L.A.N. Amaral, M. Meyer, and H.E. Stanley, Scaling of the distribution of price fluctuations of individual companies, Physical Review E 60 (1999), no. 6, 6519-6529.

[41] Peter D. Praetz, The distribution of share price changes, The Journal of Business 45 (1972), no. 1, $49-55$.

[42] K. Prause, The generalized hyperbolic model: Estimation, financial derivatives, and risk measures, Ph.D. thesis, University of Freiburg, 1999.

[43] S.D. Silvey, Statistical inference, vol. 7, Chapman \& Hall/CRC, 1975.

[44] J.A. Skjeltorp, Scaling in the Norwegian stock market, Physica A: Statistical Mechanics and its Applications 283 (2000), no. 3-4, 486-528.

[45] M.A. Stephens, Asymptotic results for goodness-of-fit statistics with unknown parameters, The Annals of Statistics (1976), 357-369.

[46] R. Weron, Computationally intensive Value at Risk calculations, Handbook of Computational Statistics, Springer, Berlin (2004), 911-950.

E-mail address: pasuarez@fis.ucm.es

E-mail address: dgullate@fis.ucm.es

Departamento de Física Teórica II, Universidad Complutense, 28040 Madrid, Spain. 